\title{
Dilute Fermi and Bose Gases
}

Subir Sachdev

\begin{abstract}
I give a unified perspective on the properties of a variety of quantum liquids using the theory of quantum phase transitions. A central role is played by a zero density quantum critical point which is argued to control the properties of the dilute gas. An exact renormalization group analysis of such quantum critical points leads to a computation of the universal properties of the dilute Bose gas and the spinful Fermi gas near a Feshbach resonance.
\end{abstract}

\section{Introduction}

This article is adapted from Chapter 16 of Quantum Phase Transitions, 2nd edition. Cambridge University Press.

It is not conventional to think of dilute quantum liquids as being in the vicinity of a quantum phase transition. However, there is a simple sense in which they are, although there is often no broken symmetry or order parameter associated with this quantum phase transition. We shall show below that the perspective of such a quantum phase transition allows a unified and efficient description of the universal properties of quantum liquids.

Stated most generally, consider a quantum liquid with a global U(1) symmetry. We shall be particularly interested in the behavior of the conserved density, generically denoted by $Q$ (usually the particle number), associated with this symmetry. The quantum phase transition is between two phases with a specific $T=0$ behavior in the expectation value of $Q$. In one of the phases, $\langle Q\rangle$ is pinned precisely at a quantized value (often zero) and does not vary as microscopic parameters are varied. This quantization ends at the quantum critical point with a discontinuity in the derivative of $\langle Q\rangle$ with respect to the tuning parameter (usually the chemical poten-

Subir Sachdev

Department of Physics, Harvard University, Cambridge MA 02138, USA

e-mail: sachdev@physics.harvard.edu 
tial), and $\langle Q\rangle$ varies smoothly in the other phase; there is no discontinuity in the value of $\langle Q\rangle$, however.

The most familiar model exhibiting such a quantum phase transition is the dilute Bose gas. We express its coherent state partition function, $Z_{B}$, in terms of complex field $\Psi_{B}(x, \tau)$, where $x$ is a $d$-dimensional spatial co-ordinate and $\tau$ is imaginary time:

$$
\begin{aligned}
& Z_{B}=\int \mathscr{D} \Psi_{B}(x, \tau) \exp \left(-\int_{0}^{1 / T} d \tau \int d^{d} x \mathscr{L}_{B}\right), \\
& \mathscr{L}_{B}=\Psi_{B}^{*} \frac{\partial \Psi_{B}}{\partial \tau}+\frac{1}{2 m}\left|\nabla \Psi_{B}\right|^{2}-\mu\left|\Psi_{B}\right|^{2}+\frac{u_{0}}{2}\left|\Psi_{B}\right|^{4} .
\end{aligned}
$$

We can identify the charge $Q$ with the boson density $\Psi_{B}^{*} \Psi_{B}$

$$
\langle Q\rangle=-\frac{\partial \mathscr{F}_{B}}{\partial \mu}=\left\langle\left|\Psi_{B}\right|^{2}\right\rangle,
$$

with $\mathscr{F}_{B}=-(T / V) \ln Z_{B}$. The quantum critical point is precisely at $\mu=0$ and $T=0$, and there are no fluctuation corrections to this location from the terms in $\mathscr{L}_{B}$. So at $T=0,\langle Q\rangle$ takes the quantized value $\langle Q\rangle=0$ for $\mu<0$, and $\langle Q\rangle>0$ for $\mu>0$; we will describe the nature of the onset at $\mu=0$ and finite- $T$ crossovers in its vicinity.

Actually, we will begin our analysis in Section 2 by a model simpler than $Z_{B}$, which displays a quantum phase transition with the same behavior in a conserved $\mathrm{U}(1)$ density $\langle Q\rangle$ and has many similarities in its physical properties. The model is exactly solvable and is expressed in terms of a continuum canonical spinless fermion field $\Psi_{F}$; its partition function is

$$
\begin{aligned}
Z_{F} & =\int \mathscr{D} \Psi_{F}(x, \tau) \exp \left(-\int_{0}^{1 / T} d \tau \int d^{d} x \mathscr{L}_{F}\right), \\
\mathscr{L}_{F} & =\Psi_{F}^{*} \frac{\partial \Psi_{F}}{\partial \tau}+\frac{1}{2 m}\left|\nabla \Psi_{F}\right|^{2}-\mu\left|\Psi_{F}\right|^{2} .
\end{aligned}
$$

$\mathscr{L}_{F}$ is just a free field theory. Like $Z_{B}, Z_{F}$ has a quantum critical point at $\mu=0$, $T=0$ and we will discuss its properties; in particular, we will show that all possible fermionic nonlinearities are irrelevant near it. The reader should not be misled by the apparently trivial nature of the model in (3); using the theory of quantum phase transitions to understand free fermions might seem like technological overkill. We will see that $Z_{F}$ exhibits crossovers that are quite similar to those near far more complicated quantum critical points, and observing them in this simple context leads to considerable insight.

In general spatial dimension, $d$, the continuum theories $Z_{B}$ and $Z_{F}$ have different, though closely related, universal properties. However, we will argue that the quantum critical points of these theories are exactly equivalent in $d=1$. We will see that the bosonic theory $Z_{B}$ is strongly coupled in $d=1$, and will note compelling evidence that the solvable fermionic theory $Z_{F}$ is its exactly universal solution in the vicinity of the $\mu=0, T=0$ quantum critical point. This equivalence extends to 
observable operators in both theories, and allows exact computation of a number of universal properties of $Z_{B}$ in $d=1$.

Our last main topic will be a discussion of the dilute spinful Fermi gas in Section 4 This generalizes $Z_{F}$ to a spin $S=1 / 2$ fermion $\Psi_{F \sigma}$, with $\sigma=\uparrow$, $\downarrow$. Now Fermi statistics do allow a contact quartic interaction, and so we have

$$
\begin{aligned}
& Z_{F s}=\int \mathscr{D} \Psi_{F \uparrow}(x, \tau) \mathscr{D} \Psi_{F \downarrow}(x, \tau) \exp \left(-\int_{0}^{1 / T} d \tau \int d^{d} x \mathscr{L}_{F s}\right), \\
& \mathscr{L}_{F s}=\Psi_{F \sigma}^{*} \frac{\partial \Psi_{F \sigma}}{\partial \tau}+\frac{1}{2 m}\left|\nabla \Psi_{F \sigma}\right|^{2}-\mu\left|\Psi_{F \sigma}\right|^{2}+u_{0} \Psi_{F \uparrow}^{*} \Psi_{F \downarrow}^{*} \Psi_{F \downarrow} \Psi_{F \uparrow} .
\end{aligned}
$$

This theory conserves fermion number, and has a phase transition as a function of increasing $\mu$ from a state with fermion number 0 to a state with non-zero fermion density. However, unlike the above two cases of $Z_{B}$ and $Z_{F}$, the transition is not always at $\mu=0$. The problem defined in (4) has recently found remarkable experimental applications in the study of ultracold gases of fermionic atoms. These experiments are aslo able to tune the value of the interaction $u_{0}$ over a wide range of values, extended from repulsive to attractive. For the attractive case, the two-particle scattering amplitude has a Feshbach resonance where the scattering length diverges, and we obtain the unitarity limit. We will see that this Feshbach resonance plays a crucial role in the phase transition obtained by changing $\mu$, and leads to a rich phase diagram of the so-called "unitary Fermi gas".

Our treatment of $Z_{F s}$ in the experimental important case of $d=3$ will show that it defines a strongly coupled field theory in the vicinity of the Feshbach resonance for attractive interactions. It therefore pays to find alternative formulations of this regime of the unitary Fermi gas. One powerful approach is to promote the two fermion bound state to a separate canonical Bose field. This yields a model, $Z_{F B}$ with both elementary fermions and bosons ; i.e. it is a combination of $Z_{B}$ and $Z_{F s}$ with interactions between the fermions and bosons. We will define $Z_{F B}$ in Section 4 , and use it to obtain a number of experimentally relevant results for the unitary Fermi gas.

Section 2 will present a thorough discussion of the universal properties of $Z_{F}$. This will be followed by an analysis of $Z_{B}$ in Section 3 , where we will use renormalization group methods to obtain perturbative predictions for universal properties. The spinful Fermi gas will be discussed in Section 4

\section{The Dilute Spinless Fermi Gas}

This section will study the properties of $Z_{F}$ in the vicinity of its $\mu=0, T=0$ quantum critical point. As $Z_{F}$ is a simple free field theory, all results can be obtained exactly and are not particularly profound in themselves. Our main purpose is to show how the results are interpreted in a scaling perspective and to obtain general lessons on the nature of crossovers at $T>0$. 
First, let us review the basic nature of the quantum critical point at $T=0$. A useful diagnostic for this is the conserved density $Q$, which in the present model we identify as $\Psi_{F}^{\dagger} \Psi_{F}$. As a function of the tuning parameter $\mu$, this quantity has a critical singularity at $\mu=0$ :

$$
\left\langle\Psi_{F}^{\dagger} \Psi_{F}\right\rangle=\left\{\begin{array}{cc}
\left(S_{d} / d\right)(2 m \mu)^{d / 2}, & \mu>0, \\
0, & \mu<0,
\end{array}\right.
$$

where the phase space factor $S_{d}=2 /\left[\Gamma(d / 2)(4 \pi)^{d / 2}\right]$.

We now proceed to a scaling analysis. Notice that at the quantum critical point $\mu=0, T=0$, the theory $\mathscr{L}_{F}$ is invariant under the scaling transformations:

$$
\begin{aligned}
x^{\prime} & =x e^{-\ell}, \\
\tau^{\prime} & =\tau e^{-z \ell}, \\
\Psi_{F}^{\prime} & =\Psi_{F} e^{d \ell / 2},
\end{aligned}
$$

provided we make the choice of the dynamic exponent

$$
z=2 .
$$

The parameter $m$ is assumed to remain invariant under the rescaling, and its role is simply to ensure that the relative physical dimensions of space and time are compatible. The transformation (6) also identifies the scaling dimension

$$
\operatorname{dim}\left[\Psi_{F}\right]=d / 2 .
$$

Now turning on a nonzero $\mu$, it is easy to see that $\mu$ is a relevant perturbation with

$$
\operatorname{dim}[\mu]=2 .
$$

There will be no other relevant perturbations at this quantum critical point, and so we have for the correlation length exponent

$$
v=1 / 2 .
$$

We can now examine the consequences of adding interactions to $\mathscr{L}_{F}$. A contact interaction such as $\int d x\left(\Psi_{F}^{\dagger}(x) \Psi_{F}(x)\right)^{2}$ vanishes because of the fermion anticommutation relation. (A contact interaction is however permitted for a spin-1/2 Fermi gas and will be discussed in Section (4) The simplest allowed term for the spinless Fermi gas is

$$
\mathscr{L}_{1}=\lambda\left(\Psi_{F}^{\dagger}(x, \tau) \nabla \Psi_{F}^{\dagger}(x, \tau) \Psi_{F}(x, \tau) \nabla \Psi_{F}(x, \tau)\right),
$$

where $\lambda$ is a coupling constant measuring the strength of the interaction. However, a simple analysis shows that

$$
\operatorname{dim}[\lambda]=-d
$$


This is negative and so $\lambda$ is irrelevant and can be neglected in the computation of universal crossovers near the point $\mu=T=0$. In particular, it will modify the result (5) only by contributions that are higher order in $\mu$.

Turning to nonzero temperatures, we can write down scaling forms. Let us define the fermion Green's function

$$
G_{F}(x, t)=\left\langle\Psi_{F}(x, t) \Psi_{F}^{\dagger}(0,0)\right\rangle ;
$$

then the scaling dimensions above imply that it satisfies

$$
G_{F}(x, t)=(2 m T)^{d / 2} \Phi_{G_{F}}\left((2 m T)^{1 / 2} x, T t, \frac{\mu}{T}\right),
$$

where $\Phi_{G_{F}}$ is a fully universal scaling function. For this particularly simple theory $\mathscr{L}_{F}$ we can of course obtain the result for $G_{F}$ in closed form:

$$
G_{F}(x, t)=\int \frac{d^{d} k}{(2 \pi)^{d}} \frac{e^{i k x-i\left(k^{2} /(2 m)-\mu\right) t}}{1+e^{-\left(k^{2} /(2 m)-\mu\right) / T}},
$$

and it is easy to verify that this obeys the scaling form 147. Similarly the free energy $\mathscr{F}_{F}$ has scaling dimension $d+z$, and we have

$$
\mathscr{F}_{F}=T^{d / 2+1} \Phi_{\mathscr{F}_{F}}\left(\frac{\mu}{T}\right)
$$

with $\Phi_{\mathscr{F}_{F}}$ a universal scaling function; the explicit result is, of course,

$$
\mathscr{F}_{F}=-\int \frac{d^{d} k}{(2 \pi)^{d}} \ln \left(1+e^{\left(\mu-k^{2} /(2 m)\right) / T}\right),
$$

which clearly obeys (16). The crossover behavior of the fermion density

$$
\langle Q\rangle=\left\langle\Psi_{F}^{\dagger} \Psi_{F}\right\rangle=-\frac{\partial \mathscr{F}_{F}}{\partial \mu}
$$

follows by taking the appropriate derivative of the free energy. Examination of these results leads to the crossover phase diagram of Fig. 11 We will examine each of the regions of the phase diagram in turn, beginning with the two low-temperature regions.

\subsection{Dilute Classical Gas, $k_{B} T \ll|\mu|, \mu<0$}

The ground state for $\mu<0$ is the vacuum with no particles. Turning on a nonzero temperature produces particles with a small nonzero density $\sim e^{-|\mu| / T}$. The de Broglie wavelength of the particles is of order $T^{-1 / 2}$, which is significantly smaller than the mean spacing between the particles, which diverges as $e^{|\mu| / d T}$ as $T \rightarrow 0$. 


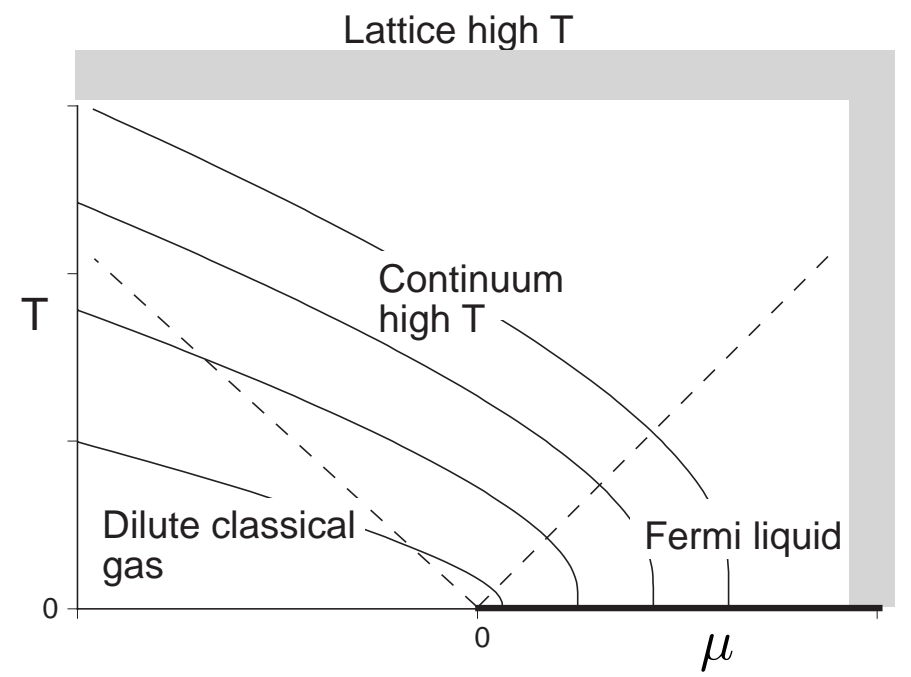

Fig. 1 Phase diagram of the dilute Fermi gas $Z_{F}$ (Eqn. (3) ) as a function of the chemical potential $\mu$ and the temperature $T$. The regions are separated by crossovers denoted by dashed lines, and their physical properties are discussed in the text. The full lines are contours of equal density, with higher densities above lower densities; the zero density line is $\mu<0, T=0$. The line $\mu>0$, $T=0$ is a line of $z=1$ critical points that controls the longest scale properties of the low- $T$ Fermi liquid region. The critical end point $\mu=0, T=0$ has $z=2$ and controls global structure of the phase diagram. In $d=1$, the Fermi liquid is more appropriately labeled a Tomonaga-Luttinger liquid. The shaded region marks the boundary of applicability of the continuum theory and occurs at $\mu, T \sim w$.

This implies that the particles behave semiclassically. To leading order from [15], the fermion Green's function is simply the Feynman propagator of a single particle

$$
G_{F}(x, t)=\left(\frac{m}{2 \pi i t}\right)^{d / 2} \exp \left(-\frac{i m x^{2}}{2 t}\right),
$$

and the exclusion of states from the other particles has only an exponentially small effect. Notice that $G_{F}$ is independent of $\mu$ and $T$ and $(19)$ is the exact result for $\mu=T=0$. The free energy, from (16) and (17), is that of a classical Boltzmann gas

$$
\mathscr{F}_{F}=-\left(\frac{m T}{2 \pi}\right)^{d / 2} e^{-|\mu| / T} .
$$

\subsection{Fermi Liquid, $k_{B} T \ll \mu, \mu>0$}

The behavior in this regime is quite complex and rich. As we will see, and as noted in Fig. 1. the line $\mu>0, T=0$ is itself a line of quantum critical points. The interplay 
between these critical points and those of the $\mu=0, T=0$ critical end point is displayed quite instructively in the exact results for $G_{F}$ and is worth examining in detail. It must be noted that the scaling dimensions and critical exponents of these two sets of critical points need not (and indeed will not) be the same. The behavior of the $\mu>0, T=0$ critical line emerges as a particular scaling limit of the global scaling functions of the $\mu=0, T=0$ critical end point. Thus the latter scaling functions are globally valid everywhere in Fig. 1 and describe the physics of all its regimes.

First it can be argued, for example, by studying asymptotics of the integral in (15), that for very short times or distances, the correlators do not notice the consequences of other particles present because of a nonzero $T$ or $\mu$ and are therefore given by the single-particle propagator, which is the $T=\mu=0$ result in (19). More precisely we have

$$
G(x, t) \text { is given by }\left[19 \text { ) for }|x| \ll(2 m \mu)^{-1 / 2}, \quad|t| \ll \frac{1}{\mu} .\right.
$$

With increasing $x$ or $t$, the restrictions in (21) are eventually violated and the consequences of the presence of other particles, resulting from a nonzero $\mu$, become apparent. Notice that because $\mu$ is much larger than $T$, it is the first energy scale to be noticed, and as a first approximation to understand the behavior at larger $x$ we may ignore the effects of $T$.

Let us therefore discuss the ground state for $\mu>0$. It consists of a filled Fermi sea of particles (a Fermi liquid) with momenta $k<k_{F}=(2 m \mu)^{1 / 2}$. An important property of the this state is that it permits excitations at arbitrarily low energies (i.e., it is gapless). These low energy excitations correspond to changes in occupation number of fermions arbitrarily close to $k_{F}$. As a consequence of these gapless excitations, the points $\mu>0(T=0)$ form a line of quantum critical points, as claimed earlier. We will now derive the continuum field theory associated with this line of critical points. We are interested here only in $x$ and $t$ values that violate the constraints in (21), and so in occupation of states with momenta near $\pm k_{F}$. So let us parameterize, in $d=1$,

$$
\Psi(x, \tau)=e^{i k_{F} x} \Psi_{R}(x, \tau)+e^{-i k_{F} x} \Psi_{L}(x, \tau),
$$

where $\Psi_{R, L}$ describe right- and left-moving fermions and are fields that vary slowly on spatial scales $\sim 1 / k_{F}=(1 / 2 m \mu)^{1 / 2}$ and temporal scales $\sim 1 / \mu$; most of the results discussed below hold, with small modifications, in all $d$. Inserting the above parameterization in $\mathscr{L}_{F}$, and keeping only terms lowest order in spatial gradients, we obtain the "effective" Lagrangean for the Fermi liquid region, $\mathscr{L}_{F L}$ in $d=1$ :

$$
\mathscr{L}_{F L}=\Psi_{R}^{\dagger}\left(\frac{\partial}{\partial \tau}-i v_{F} \frac{\partial}{\partial x}\right) \Psi_{R}+\Psi_{L}^{\dagger}\left(\frac{\partial}{\partial \tau}+i v_{F} \frac{\partial}{\partial x}\right) \Psi_{L},
$$

where $v_{F}=k_{F} / m=(2 \mu / m)^{1 / 2}$ is the Fermi velocity. Now notice that $\mathscr{L}_{F L}$ is invariant under a scaling transformation, which is rather different from (6) for the $\mu=0$, $T=0$ quantum critical point: 


$$
\begin{aligned}
x^{\prime} & =x e^{-\ell}, \\
\tau^{\prime} & =\tau e^{-\ell}, \\
\Psi_{R, L}^{\prime}\left(x^{\prime}, \tau^{\prime}\right) & =\Psi_{R, L}(x, \tau) e^{\ell / 2}, \\
v_{F}^{\prime} & =v_{F} .
\end{aligned}
$$

The above results imply

$$
z=1
$$

unlike $z=2$ (Eqn. (7)) at the $\mu=0$ critical point, and

$$
\operatorname{dim}\left[\Psi_{R, L}\right]=1 / 2,
$$

which actually holds for all $d$ and therefore differs from (8). Further notice that $v_{F}$, and therefore $\mu$, are invariant under rescaling, unlike (9) at the $\mu=0$ critical point. Thus $v_{F}$ plays a role rather analogous to that of $m$ at the $\mu=0$ critical point: It is simply the physical units of spatial and length scales. The transformations 24 show that $\mathscr{L}_{L F}$ is scale invariant for each value of $\mu$, and we therefore have a line of quantum critical points as claimed earlier. It should also be emphasized that the scaling dimension of interactions such as $\lambda$ will also change; in particular not all interactions are irrelevant about the $\mu \neq 0$ critical points. These new interactions are, however, small in magnitude provided $\mu$ is small (i.e., provided we are within the domain of validity of the global scaling forms (14) and (16), and so we will neglect them here. Their main consequence is to change the scaling dimension of certain operators, but they preserve the relativistic and conformal invariance of $\mathscr{L}_{F L}$. This more general theory of $d=1$ fermions is the Tomonaga-Luttinger liquid.

\subsection{High-T Limit, $k_{B} T \gg|\mu|$}

This is the last, and in many ways the most interesting, region of Fig. 10 Now $T$ is the most important energy scale controlling the deviation from the $\mu=0, T=0$ quantum critical point, and the properties will therefore have some similarities to the "quantum critical region" of other strongly interacting models [17]. It should be emphasized that while the value of $T$ is significantly larger than $|\mu|$, it cannot be so large that it exceeds the limits of applicability for the continuum action $\mathscr{L}_{F}$. If we imagine that $\mathscr{L}_{F}$ was obtained from a model of lattice fermions with bandwidth $w$, then we must have $T \ll w$.

We discuss first the behavior of the fermion density. In the high- $T$ limit of the continuum theory $\mathscr{L}_{F},|\mu| \ll T \ll w$, we have from (17) and (18) the universal result

$$
\left\langle\Psi_{F}^{\dagger} \Psi_{F}\right\rangle=(2 m T)^{d / 2} \int \frac{d^{d} y}{(2 \pi)^{d}} \frac{1}{e^{y^{2}}+1}
$$




$$
=(2 m T)^{d / 2} \zeta(d / 2) \frac{\left(1-2^{d / 2}\right)}{(4 \pi)^{d / 2}} .
$$

This density implies an interparticle spacing that is of order the de Broglie wavelength $=(1 / 2 m T)^{1 / 2}$. Hence thermal and quantum effects are to be equally important, and neither dominate.

For completeness, let us also consider the fermion density for $T \gg w$ (the region above the shaded region in Fig. 11, to illustrate the limitations on the continuum description discussed above. Now the result depends upon the details of the nonuniversal fermion dispersion; on a hypercubic lattice with dispersion $\varepsilon_{k}-\mu$, we obtain

$$
\begin{aligned}
\left\langle\Psi_{F}^{\dagger} \Psi_{F}\right\rangle & =\int_{-\pi / a}^{\pi / a} \frac{d^{d} k}{(2 \pi)^{d}} \frac{1}{e^{\left(\varepsilon_{k}-\mu\right) / T}+1} \\
& =\frac{1}{2 a^{d}}-\frac{1}{4 T} \int_{-\pi / a}^{\pi / a} \frac{d^{d} k}{(2 \pi)^{d}}\left(\varepsilon_{k}-\mu\right)+\mathscr{O}\left(1 / T^{2}\right) .
\end{aligned}
$$

The limits on the integration, which extend from $-\pi / a$ to $\pi / a$ for each momentum component, had previously been sent to infinity in the continuum limit $a \rightarrow 0$. In the presence of lattice cutoff, we are able to make a naive expansion of the integrand in powers of $1 / T$, and the result therefore only contains negative integer powers of $T$. Contrast this with the universal continuum result 27) where we had noninteger powers of $T$ dependent upon the scaling dimension of $\Psi$.

We return to the universal high- $T$ region, $|\mu| \ll T \ll w$, and describe the behavior of the fermionic Green's function $G_{F}$, given in (15). At the shortest scales we again have the free quantum particle behavior of the $\mu=0, T=0$ critical point:

$$
G_{F}(x, t) \text { is given by }\left[19 \text { ) for }|x| \ll(2 m T)^{-1 / 2},|t| \ll \frac{1}{T} .\right.
$$

Notice that the limits on $x$ and $t$ in (29) are different from those in (21), in that they are determined by $T$ and not $\mu$. At larger $|x|$ or $t$ the presence of the other thermally excited particles becomes apparent, and $G_{F}$ crosses over to a novel behavior characteristic of the high- $T$ region. We illustrate this by looking at the large- $x$ asymptotics of the equal-time $G$ in $d=1$ (other $d$ are quite similar):

$$
G_{F}(x, 0)=\int \frac{d k}{2 \pi} \frac{e^{i k x}}{1+e^{-k^{2} / 2 m T}} .
$$

For large $x$ this can be evaluated by a contour integration, which picks up contributions from the poles at which the denominator vanishes in the complex $k$ plane. The dominant contributions come from the poles closest to the real axis, and give the leading result

$$
G_{F}(|x| \rightarrow \infty, 0)=-\left(\frac{\pi^{2}}{2 m T}\right)^{1 / 2} \exp \left(-(1-i)(m \pi T)^{1 / 2} x\right) .
$$


Thermal effects therefore lead to an exponential decay of equal-time correlations, with a correlation length $\xi=(m \pi T)^{-1 / 2}$. Notice that the $T$ dependence is precisely that expected from the exponent $z=2$ associated with the $\mu=0$ quantum critical point and the general scaling relation $\xi \sim T^{-1 / z}$. The additional oscillatory term in 31 is a reminder that quantum effects are still present at the scale $\xi$, which is clearly of order the de Broglie wavelength of the particles.

\section{The Dilute Bose Gas}

This section will study the universal properties quantum phase transition of the dilute Bose gas model $Z_{B}$ in (1) in general dimensions. We will begin with a simple scaling analysis that will show that $d=2$ is the upper-critical dimension. The first subsection will analyze the case $d<2$ in some more detail, while the next subsection will consider the somewhat different properties in $d=3$. Some of the results of this section were also obtained by Kolomeisky and Straley [9, 10].

We begin with the analog of the simple scaling considerations presented at the beginning of Section 2 At the coupling $u=0$, the $\mu=0$ quantum critical point of $\mathscr{L}_{B}$ is invariant under the transformations (6), after the replacement $\Psi_{F} \rightarrow \Psi_{B}$, and we have as before $z=2$ and

$$
\operatorname{dim}\left[\Psi_{B}\right]=d / 2, \quad \operatorname{dim}[\mu]=2 ;
$$

these results will shortly be seen to be exact in all $d$. We can easily determine the scaling dimension of the quartic coupling $u$ at the $u=0, \mu=0$ fixed point under the bosonic analog of the transformations (6); we find

$$
\operatorname{dim}\left[u_{0}\right]=2-d .
$$

Thus the free-field fixed point is stable for $d>2$, in which case it is suspected that a simple perturbative analysis of the consequences of $u$ will be adequate. However, for $d<2$, a more careful renormalization group-based resummation of the consequences of $u$ is required. This identifies $d=2$ as the upper-critical dimension of the present quantum critical point.

Our analysis of the case $d<2$ for the dilute Bose gas quantum critical point will find, somewhat surprisingly, that all the renormalizations, and the associated flow equations, can be determined exactly in closed form. We begin by considering the one-loop renormalization of the quartic coupling $u_{0}$ at the $\mu=0, T=0$ quantum critical point. It turns out that only the ladder series of Feynman diagrams shown in Fig. 2 need be considered (the $T$ matrix). Evaluating the first term of the series in Fig. 2] for the case of zero external frequency and momenta, we obtain the contribution

$$
-u_{0}^{2} \int \frac{d \omega}{2 \pi} \int \frac{d^{d} k}{(2 \pi)^{d}} \frac{1}{\left(-i \omega+k^{2} /(2 m)\right)} \frac{1}{\left(i \omega+k^{2} /(2 m)\right)}=-u_{0}^{2} \int \frac{d^{d} k}{(2 \pi)^{d}} \frac{m}{k^{2}}
$$




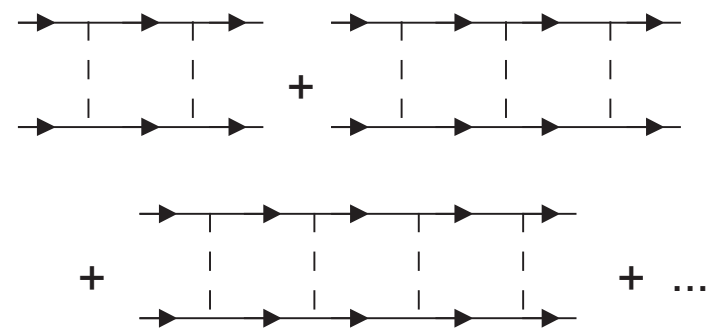

Fig. 2 The ladder series of diagrams that contribute the renormalization of the coupling $u$ in $Z_{B}$ for $d<2$.

(the remaining ladder diagrams are powers of 34 and form a simple geometric series). Notice the infrared singularity for $d<2$, which is cured by moving away from the quantum critical point, or by external momenta.

We can proceed further by a simple application of the momentum shell RG. Note that we will apply cutoff $\Lambda$ only in momentum space. The RG then proceeds by integrating all frequencies, and momentum modes in the shell between $\Lambda e^{-\ell}$ and $\Lambda$. The renormalization of the coupling $u_{0}$ is then given by the first diagram in Fig. 2. and after absorbing some phase space factors by a redefinition of interaction coupling

$$
u_{0}=\frac{\Lambda^{2-d}}{2 m S_{d}} u
$$

we obtain [6, 7]

$$
\frac{d u}{d \ell}=\varepsilon u-\frac{u^{2}}{2} .
$$

Here $S_{d}=2 /\left(\Gamma(d / 2)(4 \pi)^{d / 2}\right)$ is the usual phase space factor, and

$$
\varepsilon=2-d
$$

Note that for $\varepsilon>0$, there is a stable fixed point at

$$
u^{*}=2 \varepsilon,
$$

which will control all the universal properties of $Z_{B}$.

The flow equation $(36)$, and the fixed point value 38 are exact to all orders in $u$ or $\varepsilon$, and it is not necessary to consider $u$-dependent renormalizations to the field scale of $\Psi_{B}$ or any of the other couplings in $Z_{B}$. This result is ultimately a consequence of a very simple fact: The ground state of $Z_{B}$ at the quantum critical point $\mu=0$ is simply the empty vacuum with no particles. So any interactions that appear are entirely due to particles that have been created by the external fields. In particular, if we introduce the bosonic Green's function (the analog of (15))

$$
G_{B}(x, t)=\left\langle\Psi_{B}(x, t) \Psi_{B}^{\dagger}(0,0)\right\rangle,
$$


then for $\mu \leq 0$ and $T=0$, its Fourier transform $G(k, \omega)$ is given exactly by the free field expression

$$
G_{B}(k, \omega)=\frac{1}{-\omega+k^{2} /(2 m)-\mu} .
$$

The field $\Psi_{B}^{\dagger}$ creates a particle that travels freely until its annihilation at $(x, t)$ by the field $\Psi_{B}$; there are no other particles present at $T=0, \mu \leq 0$, and so the propagator is just the free field one. The simple result (40) implies that the scaling dimensions in (32) are exact. Turning to the renormalization of $u$, it is clear from the diagram in Fig. 2 that we are considering the interactions of just two particles. For these, the only nonzero diagrams are the one shown in Fig. 2. which involve repeated scattering of just these particles. Formally, it is possible to write down many other diagrams that could contribute to the renormalization of $u$; however, all of these vanish upon performing the integral over internal frequencies for there is always one integral that can be closed in one half of the frequency plane where the integrand has no poles. This absence of poles is of course just a more mathematical way of stating that there are no other particles around.

We will consider application of these renormalization group results separately for the cases below and above the upper-critical dimension of $d=2$.

\section{$3.1 d<2$}

First, let us note some important general implications of the theory controlled by the fixed point interaction 38 . As we have already noted, the scaling dimensions of $\Psi_{B}$ and $\mu$ are given precisely by their free field values in $\underline{32}$, and the dynamic exponent $z$ also retains the tree-level value $z=2$. All these scaling dimensions are identical to those obtained for the case of the spinless Fermi gas in Section 2 . Further, the presence of a nonzero and universal interaction strength $u^{*}$ in (38) implies that the bosonic system is stable for the case $\mu>0$ because the repulsive interactions will prevent the condensation of infinite density of bosons (no such interaction was necessary for the fermion case, as the Pauli exclusion was already sufficient to stabilize the system). These two facts imply that the formal scaling structure of the bosonic fixed point being considered here is identical to that of the fermionic one considered in Section 2 and that the scaling forms of the two theories are identical. In particular, $G_{B}$ will obey a scaling form identical to that for $G_{F}$ in 114) (with a corresponding scaling function $\Phi_{G_{B}}$ ), while the free energy, and associated derivatives, obey (16) (with a scaling function $\Phi_{\mathscr{F}_{B}}$ ). The universal functions $\Phi_{G_{B}}$ and $\Phi_{\mathscr{F}_{B}}$ can be determined order by order in the present $\varepsilon=2-d$ expansion, and this will be illustrated shortly.

Although the fermionic and bosonic fixed points share the same scaling dimensions, they are distinct fixed points for general $d<2$. However, these two fixed points are identical precisely in $d=1$ [19]. Evidence for this was presented in Ref. [5], where the anomalous dimension of the composite operator $\Psi_{B}^{2}$ was com- 
puted exactly in the $\varepsilon$ expansion and was found to be identical to that of the corresponding fermionic operator. Assuming the identity of the fixed points, we can then make a stronger statement about the universal scaling function: those for the free energy (and all its derivatives) are identical $\Phi_{\mathscr{F}_{B}}=\Phi_{\mathscr{F}_{F}}$ in $d=1$. In particular, from (17) and (18) we conclude that the boson density is given by

$$
\langle Q\rangle=\left\langle\Psi_{B}^{\dagger} \Psi_{B}\right\rangle=\int \frac{d k}{2 \pi} \frac{1}{e^{\left(k^{2} /(2 m)-\mu\right) / T}+1}
$$

in $d=1$ only. The operators $\Psi_{B}$ and $\Psi_{F}$ are still distinct and so there is no reason for the scaling functions of their correlators to be the same. However, in $d=1$, we can relate the universal scaling function of $\Psi_{B}$ to those of $\Psi_{F}$ via a continuum version of the Jordan-Wigner transformation

$$
\Psi_{B}(x, t)=\exp \left(i \pi \int_{-\infty}^{x} d y \Psi_{F}^{\dagger}(y, t) \Psi_{F}(y, t)\right) \Psi_{F}(x, t) .
$$

This identity is applied to obtain numerous exact results in Ref. [17]

As not all observables can be computed exactly in $d=1$ by the mapping to the free fermions, we will now consider the $\varepsilon=2-d$ expansion. We will present a simple $\varepsilon$ expansion calculation [18] for illustrative purposes. We focus on density of bosons at $T=0$. Knowing that the free energy obeys the analog of (16), we can conclude that a relationship like (5) holds:

$$
\left\langle\Psi_{B}^{\dagger} \Psi_{B}\right\rangle=\left\{\begin{array}{cc}
\mathscr{C}_{d}(2 m \mu)^{d / 2}, & \mu>0, \\
0, & \mu<0,
\end{array}\right.
$$

at $T=0$, with $\mathscr{C}_{d}$ a universal number. The identity of the bosonic and fermionic theories in $d=1$ implies from (5) or from (41) that $\mathscr{C}_{1}=S_{1} / 1=1 / \pi$. We will show how to compute $\mathscr{C}_{d}$ in the $\varepsilon$ expansion; similar techniques can be used for almost any observable.

Even though the position of the fixed point is known exactly in (38), not all observables can be computed exactly because they have contributions to arbitrary order in $u$. However, universal results can be obtained order-by-order in $u$, which then become a power series in $\varepsilon=2-d$. As an example, let us examine the low order contributions to the boson density. To compute the boson density for $\mu>0$, we anticipate that there is condensate of the boson field $\Psi_{B}$, and so we write

$$
\Psi_{B}(x, \tau)=\Psi_{0}+\Psi_{1}(x, t),
$$

where $\Psi_{1}$ has no zero wavevector and frequency component. Inserting this into $\mathscr{L}_{B}$ in (11), and expanding to second order in $\Psi_{1}$, we get

$$
\begin{aligned}
\mathscr{L}_{1}= & -\mu\left|\Psi_{0}\right|^{2}+\frac{u_{0}}{2}\left|\Psi_{0}\right|^{4}-\Psi_{1}^{*} \frac{\partial \Psi_{1}}{\partial \tau}+\frac{1}{2 m}\left|\nabla \Psi_{1}\right|^{2} \\
& -\mu\left|\Psi_{1}\right|^{2}+\frac{u_{0}}{2}\left(4\left|\Psi_{0}\right|^{2}\left|\Psi_{1}\right|^{2}+\Psi_{0}^{2} \Psi_{1}^{* 2}+\Psi_{0}^{* 2} \Psi_{1}^{2}\right) .
\end{aligned}
$$


This is a simple quadratic theory in the canonical Bose field $\Psi_{1}$, and its spectrum and ground state energy can be determined by the familiar Bogoliubov transformation. Carrying out this step, we obtain the following formal expression for the free energy density $\mathscr{F}$ as a function of the condensate $\Psi_{0}$ at $T=0$ :

$$
\begin{aligned}
\mathscr{F}\left(\Psi_{0}\right)= & -\mu\left|\Psi_{0}\right|^{2}+\frac{u_{0}}{2}\left|\Psi_{0}\right|^{4}+\frac{1}{2} \int \frac{d^{d} k}{(2 \pi)^{d}}\left[\left\{\left(\frac{k^{2}}{2 m}-\mu+2 u_{0}\left|\Psi_{0}\right|^{2}\right)^{2}-u_{0}^{2}\left|\Psi_{0}\right|^{4}\right\}^{1 / 2}\right. \\
& \left.-\left(\frac{k^{2}}{2 m}-\mu+2 u_{0}\left|\Psi_{0}\right|^{2}\right)\right] .
\end{aligned}
$$

To obtain the physical free energy density, we have to minimize $\mathscr{F}$ with respect to variations in $\Psi_{0}$ and to substitute the result back into (46). Finally, we can take the derivative of the resulting expression with respect to $\mu$ and obtain the required expression for the boson density, correct to the first two orders in $u_{0}$ :

$$
\left\langle\Psi_{B}^{\dagger} \Psi_{B}\right\rangle=\frac{\mu}{u_{0}}+\frac{1}{2} \int \frac{d^{d} k}{(2 \pi)^{d}}\left[1-\frac{k^{2}}{\sqrt{k^{2}\left(k^{2}+4 m \mu\right)}}\right] .
$$

To convert 47) into a universal result, we need to evaluate it at the coupling appropriate to the fixed point (38). This is most easily done by the field-theoretic RG. So let us translate the RG equation (36) into this language. We introduce a momentum scale $\tilde{\mu}$ (the tilde is to prevent confusion with the chemical potential) and express $u_{0}$ in terms of a dimensionless coupling $u_{R}$ by

$$
u_{0}=u_{R} \frac{(2 m) \tilde{\mu}^{\varepsilon}}{S_{d}}\left(1+\frac{u_{R}}{2 \varepsilon}\right) .
$$

The motivation behind the choice of the renormalization factor in (48) is that the renormalized four-point coupling, when expressed in terms of $u_{R}$, and evaluated in $d=2-\varepsilon$, is free of poles in $\varepsilon$ as can easily be explicitly checked using (34) and the associated geometric series. Then, we evaluate (47) at the fixed point value of $u_{R}$, compute any physical observable as a formal diagrammatic expansion in $u_{0}$, substitute $u_{0}$ in favor of $u_{R}$ using (48), and expand the resulting expression in powers of $\varepsilon$. All poles in $\varepsilon$ should cancel, but the resulting expression will depend upon the arbitrary momentum scale $\tilde{\mu}$. At the fixed point value $u_{R}^{*}$, dependence upon $\tilde{\mu}$ then disappears and a universal answer remains. In this manner we obtain from 477 a universal expression in the form 433 with

$$
\mathscr{C}_{d}=S_{d}\left[\frac{1}{2 \varepsilon}+\frac{\ln 2-1}{4}+\mathscr{O}(\varepsilon)\right] .
$$




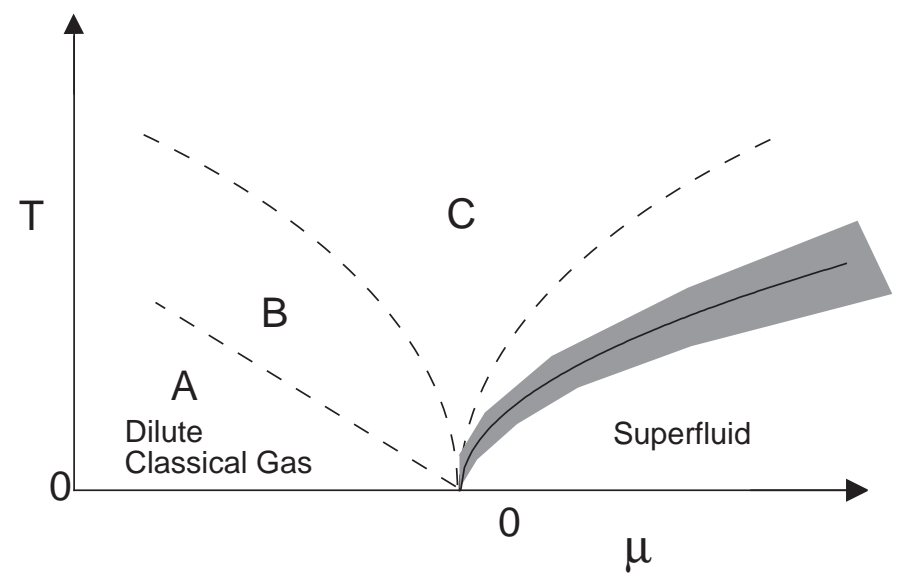

Fig. 3 Crossovers of the dilute Bose gas in $d=3$ as a function of the chemical potential $\mu$ and the temperature $T$. The regimes labeled A, B, C are described in Ref. [17]. The solid line is the finitetemperature phase transition where the superfluid order disappears; the shaded region is where there is an effective classical description of thermal fluctuations. The contours of constant density are similar to those in Fig. 1 and are not displayed.

\section{$3.2 d=3$}

Now we briefly discuss $2<d<4$ : details appear elsewhere [17]. In $d=2$, the upper critical dimension, there are logarithmic corrections which were computed by Prokof'ev et al. [15]. Related results, obtained through somewhat different methods, are available in the literature [13, 14, 6, 19].

The quantum critical point at $\mu=0, T=0$ is above its upper-critical dimension, and we expect mean-field theory to apply. The analog of the mean-field result in the present context is the $T=0$ relation for the density

$$
\left\langle\Psi_{B}^{\dagger} \Psi_{B}\right\rangle=\left\{\begin{array}{cc}
\mu / u_{0}+\cdots, & \mu>0 \\
0, & \mu<0
\end{array}\right.
$$

where the ellipses represents terms that vanish faster as $\mu \rightarrow 0$. Notice that this expression for the density is not universally dependent upon $\mu$; rather it depends upon the strength of the two-body interaction $u_{0}$ (more precisely, it can be related to the $s$-wave scattering length $a$ by $u_{0}=4 \pi a / \mathrm{m}$ ). The crossovers and phase transitions at $T>0$ are sketched in Fig. 3 These are similar to those of the spinless Fermi gas, but now there can be a phase transition within one of the regions. Explicit expressions for the crossovers [17] have been presented by Rasolt et al. [16], Weichman et al. [29] and also addressed in earlier work [22, 23, 3]. 


\section{The Dilute Spinful Fermi Gas: the Feshbach Resonance}

This section turns to the case of the spinful Fermi gas with short-range interactions; as we noted in the introduction, this is a problem which has acquired renewed importance because of the new experiments on ultracold fermionic atoms.

The partition function of the theory examined in this section was displayed in (4). The renormalization group properties of this theory in the zero density limit are identical to those the dilute Bose gas considered in Section 3 . The scaling dimensions of the couplings are the same, the scaling dimension of $\Psi_{F \sigma}$ is $d / 2$ as for $\Psi_{B}$ in (32), and the flow of the $u$ is given by (36). Thus for $d<2$, a spinful Fermi gas with repulsive interactions is described by the stable fixed point in (38).

However, for the case of spinful Fermi gas case, we can consider another regime of parameters which is of great experimental importance. We can also allow $u$ to be attractive: unlike the Bose gas case, the $u<0$ case is not immediately unstable, because the Pauli exclusion principle can stabilize a Fermi gas even with attractive interactions. Furthermore, at the same time we should also consider the physically important case with $d>2$, when $\varepsilon<0$. The distinct nature of the RG flows predicted by (36) for the two signs of $\varepsilon$ are shown in Fig. 4

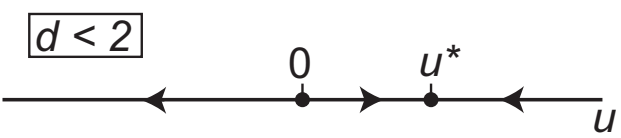

(a)

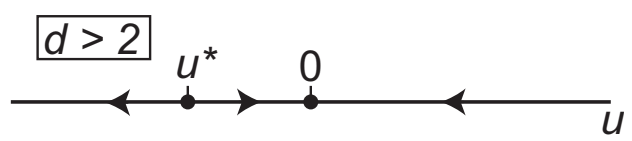

(b)

Fig. 4 The exact RG flow of 36). (a) For $d<2(\varepsilon>0)$, the infrared stable fixed point at $u=u^{*}>$ 0 describes quantum liquids of either bosons or fermions with repulsive interactions which are generically universal in the low density limit. In $d=1$ this fixed point is described by the spinless free Fermi gas ('Tonks' gas), for all statistics and spin of the constituent particles. (b) For $d>2$ $(\varepsilon<0)$ the infrared unstable fixed point at $u=u^{*}<0$ describes the Feshbach resonance which obtains for the case of attractive interactions. The relevant perturbation $\left(u-u^{*}\right)$ corresponds to the the detuning from the resonant interaction.

Notice the unstable fixed point present for $d>2$ and $u<0$. Thus accessing the fixed point requires fine-tuning of the microscopic couplings. As discussed in Refs. [12, 11], this fixed point describes a Fermi gas at a Feshbach resonance, where the interaction between the fermions is universal. For $u<u^{*}$, the flow is to $u \rightarrow-\infty$ : this corresponds to a strong attractive interaction between the fermions, which then bind into tightly bound pairs of bosons, which then Bose condense; this corresponds to the so-called 'BEC' regime. On the other hand, for $u>u^{*}$, the flow is to $u \nearrow$ 
0 , and the weakly interacting fermions then form the Bardeen-Cooper-Schrieffer (BCS) superconducting state.

Note that the fixed point at $u=u^{*}$ for $Z_{F s}$ has two relevant directions for $d>2$. As in the other problems considered earlier, one corresponds to the chemical potential $\mu$. The other corresponds to the deviation from the critical point $u-u^{*}$, and this (from (36)) has RG eigenvalue $-\varepsilon=d-2>0$. This perturbation corresponds to the "detuning" from the Feshbach resonance, $v$ (not to be confused with the symbol for the correlation length exponent); we have $v \propto u-u^{*}$. Thus we have

$$
\operatorname{dim}[\mu]=2, \operatorname{dim}[v]=d-2 .
$$

These two relevant perturbations will have important consequences for the phase diagram, as we will see shortly.

For now, let us understand the physics of the Feshbach resonance better. For this, it is useful to compute the two body $T$ matrix exactly by summing the graphs in Fig. 2, along with a direct interaction first order in $u_{0}$. The second order term was already evaluated for the bosonic case in (34) for zero external momentum and frequency, and has an identical value for the present fermionic case. Here, however, we want the off-shell $T$-matrix, for the case in which the incoming particles have momenta $k_{1,2}$, and frequencies $\omega_{1,2}$. Actual for the simple momentum-independent interaction $u_{0}$, the $T$ matrix depends only upon the sums $k=k_{1}+k_{2}$ and $\omega=\omega_{1}+$ $\omega_{2}$, and is independent of the final state of the particles, and the diagrams in Fig. 2 form a geometric series. In this manner we obtain

$$
\begin{aligned}
& \frac{1}{T(k, i \omega)}=\frac{1}{u_{0}} \\
& +\int \frac{d \Omega}{2 \pi} \int \frac{d^{d} p}{(2 \pi)^{d}} \frac{1}{\left(-i(\Omega+\omega)+(p+k)^{2} /(2 m)\right)} \frac{1}{\left(i \Omega+p^{2} /(2 m)\right)} \\
& =\frac{1}{u_{0}}+\int_{0}^{\Lambda} \frac{d^{d} p}{(2 \pi)^{d}} \frac{m}{p^{2}}+\frac{\Gamma(1-d / 2)}{(4 \pi)^{d / 2}} m^{d / 2}\left[-i \omega+\frac{k^{2}}{4 m}\right]^{d / 2-1} .
\end{aligned}
$$

In $d=3$, the $s$-wave scattering amplitude of the two particles, $f_{0}$, is related to the $T$-matrix at zero center of mass momentum and frequency $k^{2} / m$ by $f_{0}(k)=$ $-m T\left(0, k^{2} / m\right) /(4 \pi)$, and so we obtain

$$
f_{0}(k)=\frac{1}{-1 / a-i k}
$$

where the scattering length, $a$, is given by

$$
\frac{1}{a}=\frac{4 \pi}{m u_{0}}+\int_{0}^{\Lambda} \frac{d^{3} p}{(2 \pi)^{3}} \frac{4 \pi}{p^{2}}
$$

For $u_{0}<0$, we see from (54) that there is a critical value of $u_{0}$ where the scattering length diverges and changes sign: this is the Feshbach resonance. We identify this critical value with the fixed point $u=u^{*}$ of the RG flow (36). It is conventional to 
identify the deviation from the Feshbach resonance by the detuning $v$

$$
v \equiv-\frac{1}{a}
$$

Note that $v \propto u-u^{*}$, as claimed earlier. For $v>0$, we have weak attractive interactions, and the scattering length is negative. For $v<0$, we have strong attractive interactions, and a positive scattering length. Importantly, for $v<0$, there is a twoparticle bound state, whose energy can be deduced from the pole of the scattering amplitude; recalling that the reduced mass in the center of mass frame is $m / 2$, we obtain the bound state energy, $E_{b}$

$$
E_{b}=-\frac{v^{2}}{m} .
$$

We can now draw the zero temperature phase diagram [11] of $Z_{F s}$ as a function of $\mu$ and $v$, and the result is shown in Fig. 5.

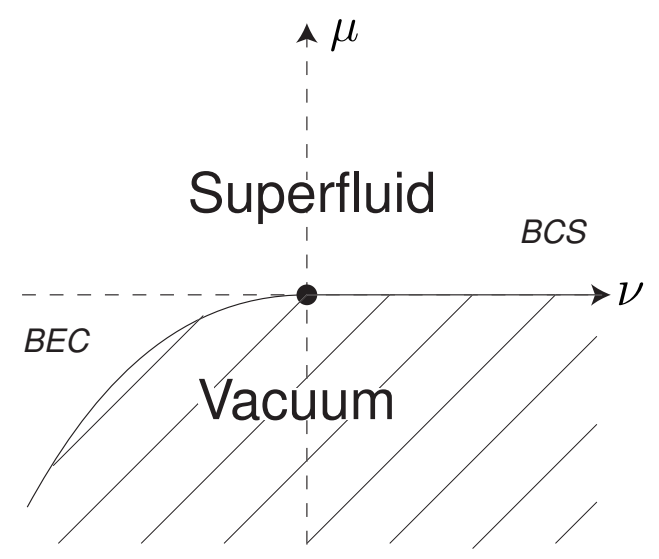

Fig. 5 Universal phase diagram at zero temperature for the spinful Fermi gas in $d=3$ as a function of the chemical potential $\mu$ and the detuning $v$. The vacuum state (shown hatched) has no particles. The position of the $v<0$ phase boundary is determined by the energy of the two-fermion bound state in 56): $\mu=-v^{2} /(2 m)$. The density of particles vanishes continuously at the second order quantum phase transition boundary of the superfluid phase, which is indicated by the thin continuous line. The quantum multicritical point at $\mu=v=0$ (denoted by the filled circle) controls all the universal physics of the dilute spinful Fermi gas near a Feshbach resonance. The universal properties of the critical line $\mu=0, v>0$ map onto the theory of Section 2 while those of the critical line $\mu=-v^{2} /(2 m), v<0$ map onto the theory of Section 3 This implies that the $T>0$ crossovers in Fig. 1 1 apply for $v>0$ (the "Fermi liquid" region of Fig. 11 now has BCS superconductivity at an exponentially small $T$ ), while those of Fig. 3 apply for $v<0$.

For $v>0$, there is no bound state, and so no fermions are present for $\mu<0$. At $\mu=0$, we have an onset of non-zero fermion density, just as in the other sections. These fermions experience a weak attractive interaction, and so experience the Cooper instability once there is a finite density of fermions for $\mu>0$. So the 
ground state for $\mu>0$ is a paired Bardeen-Cooper-Schrieffer (BCS) superfluid, as indicated in Fig. 5 For small negative scattering lengths, the BCS state modifies the fermion state only near the Fermi level. Consequently as $\mu \searrow 0$ (specifically for $\mu<v^{2} / m$ ), we can neglect the pairing in computing the fermion density. We therefore conclude that the universal critical properties of the line $\mu=0, v>0$ map precisely on to two copies (for the spin degeneracy) of the non-interacting fermion model $Z_{F}$ studied in Section 2. In particular the $T>0$ properties for $v>0$ will map onto the crossovers in Fig. 1 The only change is that the BCS pairing instability will appear below an exponentially small $T$ in the "Fermi liquid" regime. However, the scaling functions for the density as a function of $\mu / T$ will remain unchanged.

For $v<0$, the situation changes dramatically. Because of the presence of the bound state (56), it will pay to introduce fermions even for $\mu<0$. The chemical potential for a fermion pair is $2 \mu$, and so the threshold for having a non-zero density of paired fermions is $\mu=E_{b} / 2$. This leads to the phase boundary shown in Fig. 5 at $\mu=-v^{2} /(2 m)$. Just above the phase boundary, the density of fermion pairs in small, and so these can be treated as canonical bosons. Computations of the interactions between these bosons [11] show that they are repulsive. Therefore we map their dynamics onto those of the dilute Bose gas studied in Section 3 . Thus the universal properties of the critical line $\mu=-v^{2} /(2 m)$ are equivalent to those of $Z_{B}$. Specifically, this means that the $T>0$ properties across this critical line map onto those of Fig. 3 .

Thus we reach the interesting conclusion that the Feshbach resonance at $\mu=v=$ 0 is a multicritical point separating the density onset transitions of $Z_{F}$ (Section 2) and $Z_{B}$ (Section 3). This conclusion can be used to sketch the $T>0$ extension of Fig. 5, on either side of the $v=0$ line.

We now need a practical method of computing universal properties of $Z_{F s}$ near the $\mu=v=0$ fixed point, including its crossovers into the regimes described by $Z_{F}$ and $Z_{B}$. The fixed point (36) of $Z_{F s}$ provides an expansion of the critical theory in the powers of $\varepsilon=2-d$. However, observe from Fig. 4, the flow for $u<u^{*}$ is to $u \rightarrow-\infty$. The latter flow describes the crossover into the dilute Bose gas theory, $Z_{B}$, and so this cannot be controlled by the $2-d$ expansion. The following subsections will propose two alternative analyses of the Feshbach resonant fixed point which will address this difficulty.

\subsection{The Fermi-Bose Model}

One successful approach is to promote the two fermion bound state in (56) to a canonical boson field $\Psi_{B}$. This boson should also be able to mix with the scattering states of two fermions. We are therefore led to consider the following model

$$
Z_{F B}=\int \mathscr{D} \Psi_{F \uparrow}(x, \tau) \mathscr{D} \Psi_{F \downarrow}(x, \tau) \mathscr{D} \Psi_{B}(x, \tau) \exp \left(-\int d \tau d^{d} x \mathscr{L}_{F B}\right),
$$




$$
\begin{aligned}
\mathscr{L}_{F B} & =\Psi_{F \sigma}^{*} \frac{\partial \Psi_{F \sigma}}{\partial \tau}+\frac{1}{2 m}\left|\nabla \Psi_{F \sigma}\right|^{2}-\mu\left|\Psi_{F \sigma}\right|^{2} \\
& +\Psi_{B}^{*} \frac{\partial \Psi_{B}}{\partial \tau}+\frac{1}{4 m}\left|\nabla \Psi_{F \sigma}\right|^{2}+(\delta-2 \mu)\left|\Psi_{B}\right|^{2} \\
& -\lambda_{0}\left(\Psi_{B}^{*} \Psi_{F \uparrow} \Psi_{F \downarrow}+\Psi_{B} \Psi_{F \downarrow}^{*} \Psi_{F \uparrow}^{*}\right) .
\end{aligned}
$$

Here we have taken the bosons to have mass $2 m$, because that is the expected mass of the two-fermion bound state by Galilean invariance. We have omitted numerous possible quartic terms between the bosons and fermions above, and these will turn out to be irrelevant in the analysis below.

The conserved $\mathrm{U}(1)$ charge for $Z_{F B}$ is

$$
Q=\Psi_{F \uparrow}^{*} \Psi_{F \uparrow}+\Psi_{F \downarrow}^{*} \Psi_{F \downarrow}+2 \Psi_{B}^{*} \Psi_{B},
$$

and so $Z_{F B}$ is in the class of models being studied here. The factor of 2 in 58 accounts for the $2 \mu$ chemical potential for the bosons in 57). For $\mu$ sufficiently negative it is clear that $Z_{F B}$ will have neither fermions nor bosons present, and so $\langle Q\rangle=0$. Conversely for positive $\mu$, we expect $\langle Q\rangle \neq 0$, indicating a transition as a function of increasing $\mu$. Furthermore, for $\delta$ large and positive, the $Q$ density will be primarily fermions, while for $\delta$ negative the $Q$ density will be mainly bosons; thus we expect a Feshbach resonance at intermediate values of $\delta$, which then plays the role of detuning parameter.

We have thus argued that the phase diagram of $Z_{F B}$ as a function of $\mu$ and $\delta$ is qualitatively similar to that in Fig. 5, with a Feshbach resonant multicritical point near the center. The main claim of this section is that the universal properties of $Z_{F B}$ and $Z_{F s}$ are identical near this multicritical point [11, 12]. Thus, in a strong sense, the theories $Z_{F B}$ and $Z_{F s}$ are equivalent. Unlike the equivalence between $Z_{B}$ and $Z_{F}$, which held only in $d=1$, the present equivalence applies for $d>2$.

We will establish the equivalence by an exact RG analysis of the zero density critical theory. We scale the spacetime co-ordinates and the fermion field as in (6), but allow an anomalous dimension $\eta_{b}$ for the boson field relative to (32):

$$
\begin{aligned}
x^{\prime} & =x e^{-\ell}, \\
\tau^{\prime} & =\tau e^{-z \ell}, \\
\Psi_{F \sigma}^{\prime} & =\Psi_{F \sigma} e^{d \ell / 2}, \\
\Psi_{B}^{\prime} & =\Psi_{B} e^{\left(d+\eta_{b}\right) \ell / 2} \\
\lambda_{0}^{\prime} & =\lambda_{0} e^{\left(4-d-\eta_{b}\right) \ell / 2}
\end{aligned}
$$

where, as before, we have $z=2$. At tree level, the theory $Z_{F B}$ with $\mu=\delta=0$ is invariant under the transformations in 59) with $\eta_{b}=0$. At this level, we see that the coupling $\lambda_{0}$ is relevant for $d<4$, and so we will have to consider the influence of $\lambda_{0}$. This also suggests that we may be able to obtain a controlled expansion in powers of $(4-d)$. 
Upon considering corrections in powers of $\lambda_{0}$ in the critical theory, it is not difficult to show that there is a non-trivial contribution from only a single Feynman diagram: this is the self -energy diagram for $\Psi_{B}$ which is shown in Fig. 6 All other

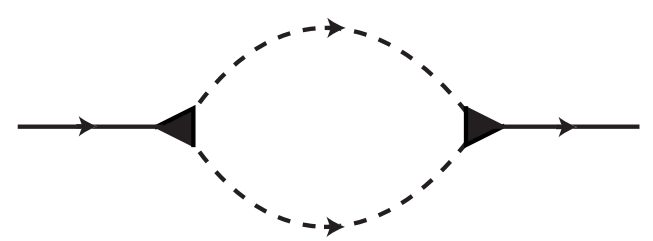

Fig. 6 Feynman diagram contributing to the RG. The dark triangle is the $\lambda_{0}$ vertex, the full line is the $\Psi_{B}$ propagator, and the dashed line is the $\Psi_{F}$ propagator.

diagrams vanish in the zero density theory, for reasons similar to those discussed for $Z_{B}$ below (38). This diagram is closely related to the integrals in the $T$-matrix computation in (52), and leads to the following contribution to the boson self energy $\Sigma_{B}$ :

$$
\begin{aligned}
& \Sigma_{B}(k, i \omega) \\
& =\lambda_{0}^{2} \int^{\frac{d}{2 \pi}} \int_{\Lambda e^{-\ell}}^{\Lambda} \frac{d^{d} p}{(2 \pi)^{d}} \frac{1}{\left(-i(\Omega+\omega)+(p+k)^{2} /(2 m)\right)} \frac{1}{\left(i \Omega+p^{2} /(2 m)\right)} \\
& =\lambda_{0}^{2} \int_{\Lambda e^{-\ell}}^{\Lambda} \frac{d^{d} p}{(2 \pi)^{d}} \frac{1}{\left(-i \omega+(p+k)^{2} /(2 m)+p^{2} /(2 m)\right)} \\
& =\lambda_{0}^{2} \int_{\Lambda e^{-\ell}}^{\Lambda} \frac{d^{d} p}{(2 \pi)^{d}} \frac{m}{p^{2}}-\lambda_{0}^{2}\left(-i \omega+\frac{k^{2}}{4 m}\left(2-\frac{4}{d}\right)\right) \int_{\Lambda e^{-\ell}}^{\Lambda} \frac{d^{d} p}{(2 \pi)^{d}} \frac{m^{2}}{p^{4}} .
\end{aligned}
$$

The first term is a constant that can absorbed into a redefinition of $\delta$. For the first time, we see above a special role for the spatial dimension $d=4$, where the momentum integral is logarithmic. Our computations below will turn to be an expansion in powers of $(4-d)$, and so we will evaluate the numerical prefactors in (60) with $d=4$. The result turns out to be correct to all orders in $(4-d)$, but to see this explicitly we need to use a proper Galilean-invariant cutoff in a field theoretic approach [11]. The simple momentum shell method being used here preserves Galilean invariance only in $d=4$.

With the above reasoning, we see that the second term in the boson self-energy in (60) can be absorbed into a rescaling of the boson field under the RG. We therefore find a non-zero anomalous dimension

$$
\eta_{b}=\lambda^{2}
$$

where we have absorbed phase space factors into the coupling $\lambda$ by

$$
\lambda_{0}=\frac{\Lambda^{2-d / 2}}{m \sqrt{S_{d}}} \lambda
$$


With this anomalous dimension, we use 59) to obtain the exact RG equation for $\lambda$ :

$$
\frac{d \lambda}{d \ell}=\frac{(4-d)}{2} \lambda-\frac{\lambda^{3}}{2} .
$$

For $d<4$, this flow has a stable fixed point at $\lambda=\lambda^{*}=\sqrt{(4-d)}$. The central claim of this subsection is that the theory $Z_{F B}$ at this fixed point is identical to the theory $Z_{F s}$ at the fixed point $u=u^{*}$ for $2<d<4$.

Before we establish this claim, note that at the fixed point, we obtain the exact result for the anomalous dimension of the the boson field

$$
\eta_{b}=4-d
$$

Let us now consider the spectrum of relevant perturbations to the $\lambda=\lambda^{*}$ fixed point. As befits a Feshbach resonant fixed point, there are 2 relevant perturbations in $Z_{F B}$, the detuning parameter $\delta$ and the chemical potential $\mu$. Apart from the tree level rescalings, at one loop we have the diagram shown in Fig. 7. This diagram has

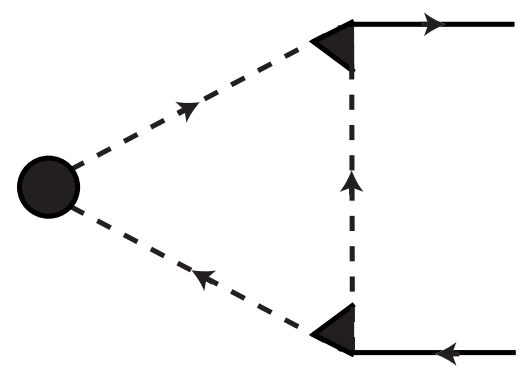

Fig. 7 Feynman diagram for the mixing between the renormalization of the $\Psi_{F}^{\dagger} \Psi_{F}$ and $\Psi_{B}^{\dagger} \Psi_{B}$ operators. The filled circle is the $\Psi_{F}^{\dagger} \Psi_{F}$ source. Other notation is as in Fig. 6

a $\Psi_{F \sigma}^{\dagger} \Psi_{F \sigma}$ source, and it renormalizes the co-efficient of $\Phi^{\dagger} \Phi$; it evaluates to

$$
\begin{aligned}
& 2 \lambda_{0}^{2} \int \frac{d \Omega}{2 \pi} \int_{\Lambda e^{-\ell}}^{\Lambda} \frac{d^{d} p}{(2 \pi)^{d}} \frac{1}{\left(-i \Omega+p^{2} /(2 m)\right)^{2}\left(i \Omega+p^{2} /(2 m)\right)} \\
& \quad=2 \lambda_{0}^{2} \int_{\Lambda e^{-\ell}}^{\Lambda} \frac{d^{d} p}{(2 \pi)^{d}} \frac{m^{2}}{p^{4}} .
\end{aligned}
$$

Combining 65 with the tree-level rescalings, we obtain the RG flow equations

$$
\begin{gathered}
\frac{d \mu}{d \ell}=2 \mu \\
\frac{d}{d \ell}(\delta-2 \mu)=\left(2-\eta_{b}\right)(\delta-2 \mu)-2 \lambda^{2} \mu
\end{gathered}
$$


where the last term arises from (65). With the value of $\eta_{b}$ in (61), the second equation simplifies to

$$
\frac{d \delta}{d \ell}=\left(2-\lambda^{2}\right) \delta
$$

Thus we see that $\mu$ and $\delta$ are actually eigen-perturbations of the fixed point at $\lambda=$ $\lambda^{*}$, and their scaling dimensions are

$$
\operatorname{dim}[\mu]=2, \operatorname{dim}[\delta]=d-2 .
$$

Note that these eigenvalues coincide with those of $Z_{F s}$ in 51 , with $\delta$ identified as proportional to the detuning $v$. This, along with the symmetries of $Q$ conservation and Galilean invariance, establishes the equivalence of the fixed points of $Z_{F B}$ and $Z_{F s}$.

The utility of the present $Z_{F B}$ formulation is that it can provide a description of universal properties of the unitary Fermi gas in $d=3$ via an expansion in $(4-d)$. Further details of explicit computations can be found in Ref. [12].

\subsection{Large $N$ expansion}

We now return to the model $Z_{F s}$ in (4), and examine it in the limit of a large number of spin components [11, 27]. We also use the structure of the large $N$ perturbation theory to obtain exact results relating different experimental observable of the unitary Fermi gas.

The basic idea of the large $N$ expansion is to endow the fermion with an additional flavor index $a=1 \ldots N / 2$, to the fermion field is $\Psi_{F \sigma a}$, where we continue to have $\sigma=\uparrow, \downarrow$. Then, we write $Z_{F s}$ as

$$
\begin{gathered}
Z_{F s}=\int \mathscr{D}_{F \sigma a}(x, \tau) \exp \left(-\int_{0}^{1 / T} d \tau \int d^{d} x \mathscr{L}_{F s}\right), \\
\mathscr{L}_{F s}=\Psi_{F \sigma a}^{*} \frac{\partial \Psi_{F \sigma a}}{\partial \tau}+\frac{1}{2 m}\left|\nabla \Psi_{F \sigma a}\right|^{2}-\mu\left|\Psi_{F \sigma a}\right|^{2} \\
+\frac{2 u_{0}}{N} \Psi_{F \uparrow a}^{*} \Psi_{F \downarrow a}^{*} \Psi_{F \downarrow b} \Psi_{F \uparrow b} .
\end{gathered}
$$

where there is implied sum over $a, b=1 \ldots N / 2$. The case of interest has $N=2$, but we will consider the limit of large even $N$, where the problem becomes tractable.

As written, there is an evident $\mathrm{O}(N / 2)$ symmetry in $Z_{F s}$ corresponding to rotations in flavor space. In addition, there is $\mathrm{U}(1)$ symmetry associated with $Q$ conservation, and a $\mathrm{SU}(2)$ spin rotation symmetry. Actually, the spin and flavor symmetry combine to make the global symmetry $\mathrm{U}(1) \times \mathrm{Sp}(N)$, but we will not make much use of this interesting observation.

The large $N$ expansion proceeds by decoupling the quartic term in 69 by a Hubbard-Stratanovich transformation. For this we introduce a complex bosonic field 
$\Psi_{B}(x, \tau)$ and write

$$
\begin{aligned}
Z_{F s}= & \int \mathscr{D} \Psi_{F \sigma a}(x, \tau) \mathscr{D} \Psi_{B}(x, \tau) \exp \left(-\int_{0}^{1 / T} d \tau \int d^{d} x \widetilde{\mathscr{L}}_{F s}\right), \\
\widetilde{\mathscr{L}_{F s}}= & \Psi_{F \sigma a}^{*} \frac{\partial \Psi_{F \sigma a}}{\partial \tau}+\frac{1}{2 m}\left|\nabla \Psi_{F \sigma a}\right|^{2}-\mu\left|\Psi_{F \sigma a}\right|^{2} \\
& +\frac{N}{2\left|u_{0}\right|}\left|\Psi_{B}\right|^{2}-\Psi_{B} \Psi_{F \uparrow a}^{*} \Psi_{F \downarrow a}^{*}-\Psi_{B}^{*} \Psi_{F \downarrow a} \Psi_{F \uparrow a} .
\end{aligned}
$$

Here, and below, we assume $u_{0}<0$, which is necessary for being near the Feshbach resonance. Note that $\Psi_{B}$ couples to the fermions just like the boson field in the BoseFermi model in [57, which is the reason for choosing this notation. If we perform the integral over $\Psi_{B}$ in (70), we recover (69), as required. For the large $N$ expansion, we have to integrate over $\Psi_{F \sigma a}$ first and obtain an effective action for $\Psi_{B}$. Because the action in (70) is Gaussian in the $\Psi_{F \sigma a}$, the integration over the fermion field involves evaluation of a functional determinant, and has the schematic form

$$
\mathscr{Z}_{F s}=\int \mathscr{D} \Psi_{B}(x, \tau) \exp \left(-N \mathscr{S}_{\text {eff }}\left[\Psi_{B}(x, \tau)\right]\right),
$$

where $\mathscr{S}_{\text {eff }}$ is the logarithm of the fermion determinant of a single flavor. The key point is that the only $N$ dependence is in the prefactor in (71), and so the theory of $\Psi_{B}$ can controlled in powers of $1 / N$.

We can expand $\mathscr{S}_{\text {eff }}$ in powers of $\Psi_{B}$ : the $p$ 'th term has a fermion loop with $p$ external $\Psi_{B}$ insertions. Details can be found in Refs. [11, 27]. Here, we only note that the expansion to quadratic order at $\mu=\delta=T=0$, in which case the co-efficient is precisely the inverse of the fermion $T$-matrix in (52):

$$
\mathscr{S}_{\text {eff }}\left[\Psi_{B}(x, \tau)\right]=-\frac{1}{2} \int \frac{d \omega}{2 \pi} \frac{d^{d} k}{(2 \pi)^{d}} \frac{1}{T(k, i \omega)}\left|\Psi_{B}(k, \omega)\right|^{2}+\ldots
$$

Given $S_{\text {eff }}$, we then have to find its saddle point with respect to $\Psi_{B}$. At $T=0$, we will find the optimal saddle point at a $\Psi_{B} \neq 0$ in the region of Fig. 5 with a non-zero density: this means that the ground state is always a superfluid of fermion pairs. The traditional expansion about this saddle point yields the $1 / N$ expansion, and many experimental observables have been computed in this manner [11, 27, 28].

We conclude our discussion of the unitary Fermi gas by deriving an exact relationship between the total energy, $E$, and the momentum distribution function, $n(k)$, of the fermions [25, 26]. We will do this using the structure of the large $N$ expansion. However, we will drop the flavor index $a$ below, and quote results directly for the physical case of $N=2$. As usual, we define the momentum distribution function by

$$
n(k)=\left\langle\Psi_{F \sigma}^{\dagger}(k, t) \Psi_{F \sigma}(k, t)\right\rangle,
$$

with no implied sum over the spin label $\sigma$. The Hamiltonian of the system in 69 is the sum of kinetic and interaction energies: the kinetic energy is clearly an integral 
over $n(k)$ and so we can write

$$
\begin{aligned}
E & =2 V \int \frac{d^{d} k}{(2 \pi)^{d}} \frac{k^{2}}{2 m} n(k)+u_{0} V\left\langle\Psi_{F \uparrow}^{\dagger} \Psi_{F \downarrow}^{\dagger} \Psi_{F \downarrow} \Psi_{F \uparrow}\right\rangle \\
& =2 V \int \frac{d^{d} k}{(2 \pi)^{d}} \frac{k^{2}}{2 m} n(k)-u_{0} \frac{\partial \ln Z_{F s}}{\partial u_{0}} .
\end{aligned}
$$

where $V$ is the system volume, and all the $\Psi_{F}$ fields are at the same $x$ and $t$. Now let us evaluate the $u_{0}$ derivative using the expression for $Z_{F s}$ in 70 ; this leads to

$$
\frac{E}{V}=2 \int \frac{d^{d} k}{(2 \pi)^{d}} \frac{k^{2}}{2 m} n(k)+\frac{1}{u_{0}}\left\langle\Psi_{B}^{*}(x, t) \Psi_{B}(x, t)\right\rangle .
$$

Now using the expression (54) relating $u_{0}$ to the scattering length $a$ in $d=3$, we can write this expression as

$$
\frac{E}{V}=\frac{m}{4 \pi a}\left\langle\Psi_{B}^{*} \Psi_{B}\right\rangle+2 \int \frac{d^{3} k}{(2 \pi)^{3}} \frac{k^{2}}{2 m}\left(n(k)-\frac{\left\langle\Psi_{B}^{*} \Psi_{B}\right\rangle m^{2}}{k^{4}}\right)
$$

This is the needed universal expression for the energy, expressed in terms of $n(k)$ and the scattering length, and independent of the short distance structure of the interactions.

At this point, it is useful to introduce "Tan's constant" $C$, defined by [25, 26]

$$
C=\lim _{k \rightarrow \infty} k^{4} n(k) .
$$

The requirement that the momentum integral in 76 is convergent in the ultraviolet implies that the limit in (77) exists, and further specifies its value

$$
C=m^{2}\left\langle\Psi_{B}^{*} \Psi_{B}\right\rangle
$$

We now note that the relationship $n(k) \rightarrow m^{2}\left\langle\Psi_{B}^{*} \Psi_{B}\right\rangle / k^{4}$ at large $k$ is also as expected from a scaling perspective. We saw in Section 4.1 that the fermion field $\Psi_{F}$ does not acquire any anomalous dimensions, and has scaling dimension $d / 2$. Consequently $n(k)$ has scaling dimension zero. Next, note that the operator $\Psi_{B}^{*} \Psi_{B}$ is conjugate to the detuning from the Feshbach critical point; from (68) the detuning has scaling dimension $d-2$, and so $\Psi_{B}^{*} \Psi_{B}$ has scaling dimension $d+z-(d-2)=4$. Combining these scaling dimensions, we explain the $k^{-4}$ dependence of $n(k)$.

It now remains to establish the claimed exact relationship in (78) as a general property of a spinful Fermi gas near unitarity. As a start, we can examine the large $k$ limit of $n(k)$ in the BCS mean field theory of the superfluid phase: the reader can easily verify that the text-book BCS expressions for $n(k)$ do indeed satisfy (78). However, the claim of Refs. [1, 24] is that (78) is exact beyond mean field theory, and also holds in the non-superfluid states at non-zero temperatures. A general proof was given in Refs. [24], and relied on the operator product expansion (OPE) applied to the field theory (70). The OPE is a general method for describing the short distance 
and time (or large momentum and frequency) behavior of field theories. Typically, in the Feynman graph expansion of a correlator, the large momentum behavior is dominated by terms in which the external momenta flow in only a few propagators, and the internal momentum integrals can be evaluated after factoring out these favored propagators. For the present situation, let us consider the $1 / N$ correction to the fermion Green's function given by the diagram in Fig. 8. Representing the bare

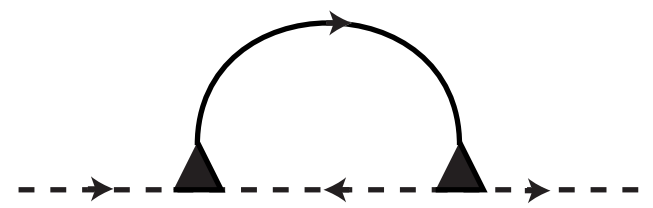

Fig. 8 Order $1 / N$ correction to the fermion Green's function. Notation is as in Fig.6

fermion and boson Green's functions by $G_{F}$ and $G_{B}$ respectively, Fig 8 evaluates to

$$
G_{F}^{2}(k, \omega) \int \frac{d^{d} p}{(2 \pi)^{d}} \frac{d \Omega}{2 \pi} G_{B}(p, \Omega) G_{F}(-k+p,-\omega+\Omega) .
$$

Here $G_{B}$ is the propagator of the boson action $\mathscr{S}_{\text {eff }}$ specified by (72). In the limit of large $k$ and $\omega$, the internal $p$ and $\Omega$ integrals are dominated by $p$ and $\Omega$ much smaller than $k$ and $\omega$; so we can approximate 79 by

$$
\begin{aligned}
& G_{F}^{2}(k, \omega) G_{F}(-k,-\omega) \int \frac{d^{d} p}{(2 \pi)^{d}} \frac{d \Omega}{2 \pi} G_{B}(p, \Omega) \\
& =G_{F}^{2}(k, \omega) G_{F}(-k,-\omega)\left\langle\Psi_{B}^{*} \Psi_{B}\right\rangle .
\end{aligned}
$$

This analysis can now be extended to all orders in $1 / N$. Among these higher order contributions are terms which contribute self energy corrections to the boson propagator $G_{B}$ in (80): it is clear that these can be summed to replace the bare $G_{B}$ in (80) by the exact $G_{B}$. Then the value of $\left\langle\left|\Psi_{B}\right|^{2}\right\rangle$ in (80) also becomes the exact value. All remaining contributions can be shown [24] to fall off faster at large $k$ and $\omega$ than the terms in 800 . So $(80)$ is the exact leading contribution to the fermion Green's function in the limit of large $k$ and $\omega$ after replacing $\left\langle\left|\Psi_{B}\right|^{2}\right\rangle$ by its exact value. We can now integrate (80) over $\omega$ to obtain $n(k)$ at large $k$. Actually the $\omega$ integral is precisely that in 65], which immediately yields the needed relation (78).

Similar analyses can be applied to determine the the spectral functions of other observables [28, 8, 20, 21, 4, 2, 24].

Determining of the specific value of Tan's constant requires numerical computations in the $1 / N$ expansion of 711. From the scaling properties of the Feshbach resonant fixed point in $d=3$, we can deduce the result obeys a scaling form similar to (14):

$$
C=(2 m T)^{2} \Phi_{C}\left(\frac{\mu}{T}, \frac{v}{\sqrt{2 m T}}\right)
$$


where $\Phi_{C}$ is a dimensionless universal function of its dimensionless arguments; note that the arguments represent the axes of Fig. 5. The methods of Refs [11, 27] can now be applied to $(78)$ to obtain numerical results for $\Phi_{C}$ in the $1 / N$ expansion. We illustrate this method here by determining $C$ to leading order in the $1 / N$ expansion at $\mu=v=0$. For this, we need to generalize the action (72) for $\Psi_{B}$ to $T>0$ and general $N$. Using (52) we can modify (72) to

$$
\mathscr{S}_{\text {eff }}=N T \sum_{\omega_{n}} \int \frac{d^{3} k}{8 \pi^{3}}\left[D_{0}\left(k, \omega_{n}\right)+D_{1}\left(k, \omega_{n}\right)\right]\left|\Psi_{B}\left(k, \omega_{n}\right)\right|^{2},
$$

where $D_{0}$ is the $T=0$ contribution, and $D_{1}$ is the correction at $T>0$ :

$$
\begin{aligned}
& D_{0}\left(k, \omega_{n}\right)=\frac{m^{3 / 2}}{16 \pi} \sqrt{-i \omega_{n}+\frac{k^{2}}{4 m}} \\
& D_{1}\left(k, \omega_{n}\right)=\frac{1}{2} \int \frac{d^{3} p}{8 \pi^{3}} \frac{1}{\left(e^{p^{2} /(2 m T)}+1\right)} \frac{1}{\left(-i \omega+p^{2} /(2 m)+(p+k)^{2} /(2 m)\right)} .
\end{aligned}
$$

We now have to evaluate $\left\langle\Psi_{B}^{*} \Psi_{B}\right\rangle$ using the Gaussian action in (82). It is useful to do this by separating the $D_{0}$ contribution, which allows us to properly deal with the large frequency behavior. So we can write

$$
\left\langle\Psi_{B}^{*} \Psi_{B}\right\rangle=\frac{1}{N} T \sum_{\omega_{n}} \int \frac{d^{3} k}{8 \pi^{3}}\left[\frac{1}{D_{0}\left(k, \omega_{n}\right)+D_{1}\left(k, \omega_{n}\right)}-\frac{1}{D_{0}\left(k, \omega_{n}\right)}\right]+D_{00}
$$

In evaluating $D_{00}$ we have to use the usual time-splitting method to ensure that the bosons are normal-ordered, and evaluate the frequency summation by analytically continuing to the real axis:

$$
\begin{aligned}
D_{00} & =\frac{1}{N} \int \frac{d^{3} k}{8 \pi^{3}} \lim _{\eta \rightarrow 0} T \sum_{\omega_{n}} \frac{e^{i \omega_{n} \eta}}{D_{0}\left(k, \omega_{n}\right)} \\
& =\frac{16 \pi}{N m^{3 / 2}} \int \frac{d^{3} k}{8 \pi^{3}} \int_{\frac{k^{2}}{4 m}}^{\infty} \frac{d \Omega}{\pi} \frac{1}{\left(e^{\Omega / T}-1\right)} \frac{1}{\sqrt{\Omega-k^{2} /(4 m)}} . \\
& =\frac{8.37758}{N} T^{2}
\end{aligned}
$$

The frequency summation in (84) can be evaluated directly on the imaginary frequency axis: the series is convergent at large $\omega_{n}$, and is easily evaluated by a direct numerical summation. Numerical evaulation of (84) now yields

$$
C=(2 m T)^{2}\left(\frac{0.67987}{N}+\mathscr{O}\left(1 / N^{2}\right)\right)
$$

at $\mu=v=0$. 


\section{References}

1. Braaten, E., and Platter, L. (2008) Phys. Rev. Lett. 100, 205301.

2. Braaten, E., Kang, D., and Platter, L. (2010) arXiv:1001.4518

3. Creswick, R. J., and Wiegel, F. W. (1983) Phys. Rev. A 28, 1579.

4. Combescot, R., Alzetto, F., and Leyronas, X. (2009) Phys. Rev. A 79, 053640

5. Damle, K., and Sachdev, S. (1996) Phys. Rev. Lett. 76, 4412.

6. Fisher, D. S., and Hohenberg, P. C. (1988) Phys. Rev. B 37, 4936.

7. Fisher, M. P. A., Weichman, P. B., Grinstein, G., and Fisher, D. S. (1989) Phys. Rev. B 40, 546.

8. Haussmann, R., Punk, M., and Zwerger, W. (2009) Phys. Rev. A 80, 063612.

9. Kolomeisky, E. B., and Straley, J. P. (1992) Phys. Rev. B 46, 11749.

10. Kolomeisky, E. B., and Straley, J. P. (1992) Phys. Rev. B 46, 13942.

11. Nikolic, P., and Sachdev, S. (2007) Phys. Rev. A 75, 033608.

12. Nishida, Y., and Son, D. T. (2007) Phys. Rev. A 75, 063617.

13. Popov, V. N. (1972) Teor. Mat. Phys. 11, 354.

14. Popov, V. N. (1983) Functional Integrals in Quantum Field Theory and Statistical Physics (D. Reidel, Dordrecht).

15. Prokof'ev, N., Ruebenacker, O., and Svistunov, B. (2001) Phys. Rev. Lett. 87, 270402.

16. Rasolt, M., Stephen, M. J., Fisher, M. E., and Weichman, P. B. (1984) Phys. Rev. Lett. 53, 798.

17. Sachdev, S. Quantum Phase Transitions, Second Edition Cambridge University Press (2011).

18. Sachdev, S., and Senthil, T. (1996) Annals of Physics 251, 76.

19. Sachdev, S., Senthil, T., and Shankar, R. (1994) Phys. Rev. B 50, 258.

20. Schneider, W., Shenoy, V. B., and Randeria, M. (2009) arXiv:0903.3006

21. Schneider, W., and Randeria, M. (2009) arXiv:0910.2693

22. Singh, K. K. (1975) Phys. Rev. B 12, 2819.

23. Singh, K. K. (1978) Phys. Rev. B 17, 324.

24. Son, D. T., and Thompson, E. G. (2010) arXiv:1002.0922

25. Tan, S. (2008) Annals of Physics 323, 2952.

26. Tan, S. (2008) Annals of Physics 323, 2971.

27. Veillette, M. Y., Sheehy, D. E., and Radzihovsky, L. (2007) Phys. Rev. A 75, 043614.

28. Veillette, M. Y., Moon, E. G., Lamacraft, A., Radzihovsky, L., Sachdev, S., and Sheehy, D. E. (2008) Phys. Rev. A 78, 033614.

29. Weichman, P. B., Rasolt, M., Fisher, M. E., and Stephen, M. J. (1986) Phys. Rev. B 33, 4632. 